\title{
Insulina: Efeitos Cardiovasculares e Aplicações Terapêuticas
}

\section{RESUMO}

Vários estudos têm sugerido benefício do uso de insulina após o infarto do miocárdio e em pacientes criticamente doentes, diabéticos e não diabéticos. No entanto, não se estabeleceu rotineiramente 0 uso das infusões de insulina e glicose, pela ausência de estudos randomizados de grande porte, entendimento precário dos mecanismos pelos quais estas infusões seriam efetivas, complexidade para administrá-las e principalmente pela mudança cultural que é exigida dos profissionais de saúde para aplicá-las na sua prática. A insulina tem efeitos benéficos no coração, tais como a otimização do uso de substratos pelos cardiomiócitos, o aumento do fluxo coronariano, efeito anti-inflamatório e também ações diretas anti-apoptóticas nas células miocárdicas. Dentro deste contexto, são revisados os resultados clínicos das infusões de insulina e glicose após infarto e cirurgia cardíaca, possíveis mecanismos fisiopatológicos responsáveis por estes benefícios e, finalmente, uma proposta de um protocolo padrão para o uso em unidades de terapia intensiva e de pós-operatório de cirurgia cardíaca. (Arq Bras Endocrinol Metab 2004;48/6:793-802)

Descritores: Insulina; Efeitos cardiovasculares; Tratamento; Hiperglicemia

\begin{abstract}
Insulin: Cardiovascular Effects and Therapeutic Implications.

Several studies have suggested a beneficial role of insulin and glucose infusions after myocardial infarction and in critically ill diabetic and nondiabetic patients. However, the insulin-glucose infusions are not routinely given to these patients, since no large randomized control trial was carried out, mechanisms involved in the benefits obtained are poorly understood, infusion protocols are complex and finally, health professionals need to change their cultural premises to apply them in their practice. Insulin has beneficial cardiovascular effects, such as the optimization of substrate use by cardiomyocites, coronary vasodilation, anti-inflammatory action and direct anti-apoptotic effects on myocardial cells. In this context, clinical studies of glucose and insulin infusions after myocardial infarction and cardiovascular surgery are reviewed, as well as possible pathophysiologic mechanisms leading to these benefits, and finally, a practical algorithm is proposed for use in intensive care and cardiovascular postoperative care units. (Arq Bras Endocrinol Metab 2004;48/6:793-802)
\end{abstract}

Keywords: Insulin; Cardiovascular effects; Treatment; Hyperglycemia

$\mathrm{O}$ CONCEITO DE QUE uma solução contendo glicose, insulina e potássio (GIK) poderia proteger os cardiomiócitos dos efeitos da isquemia foi inicialmente introduzido por Sodi-Pallares em 1962 (1). O racional para o uso desta terapia foi posteriormente formulado por Opie, em 1970, que revisão

\author{
Beatriz D. Schaan \\ Eneida R. Rabelo \\ Maria Cláudia Irigoyen
}

\begin{abstract}
Serviço de Medicina Experimental, Unidade de Pesquisa do Instituto de Cardiologia do Rio Grande do Sul e Fundação Universitária de Cardiologia (BDS), RS; Serviço de Enfermagem em Terapia Intensiva e Serviço de Cardiologia, Hospital de Clinicas de Porto Alegre (ERR), RS; e Laboratório de Hipertensão Experimental (MCI), Unidade de Hipertensão (InCor), Faculdade de Medicina da Universidade de São Paulo, SP.
\end{abstract}


sugeriu dois mecanismos principais para estes efeitos benéficos: promoção de glicólise cardíaca e desvio dos ácidos graxos livres (AGL) para os adipócitos, com resultante redução de utilização de AGL pelas células miocárdicas (2). Desde então, seguiram-se vários estudos clínicos utilizando a solução de GIK, com resultados promissores. Uma meta-análise de 9 ensaios realizados na "era pré-reperfusão", reuniu dados de 1932 pacientes randomizados ao tratamento com GIK ou não, demonstrando que seu uso teria papel importante na redução da mortalidade intra-hospitalar em indivíduos com infarto agudo do miocárdio (IAM), salvando até 49 vidas adicionais para cada 1000 pacientes (3).

Já na "era da reperfusão miocárdica", o estudo randomizado ECLA (Estudios Cardiologicos de Latinoamerica) demonstrou uma redução de $66 \%$ na mortalidade intra-hospitalar após IAM ao utilizar a solução de GIK com doses mais elevadas de insulina (4). $\mathrm{O}$ uso de doses mais altas de insulina baseou-se nos estudos de Rackley e cols., que buscaram avaliar quais seriam as taxas de infusão de GIK que poderiam determinar a máxima supressão dos AGL, assim como a máxima utilização de glicose pelos cardiomiócitos (5).

Neste mesmo período, dois outros estudos foram realizados, um com resultados negativos, mas utilizando dose baixa de insulina na solução (6), e outro avaliando apenas indivíduos com diabetes mellitus (DM) (7). Neste estudo (DIGAMI) somente com pacientes diabéticos, foram randomizados 620 indivíduos com IAM para uso de infusão contínua de insulina e glicose nas $24 \mathrm{~h}$ imediatas à admissão na unidade de tratamento intensivo ou tratamento convencional, seguindo-se, no primeiro grupo, tratamento metabólico intensivo com insulina subcutânea após a alta hospitalar. A mortalidade dos pacientes que receberam insulina e glicose foi reduzida em $30 \%$ no primeiro ano de acompanhamento (7). O seguimento destes mesmos pacientes por mais 2,5 anos (total 3,5 anos) demonstrou um melhor prognóstico também a longo prazo, obtendo-se redução absoluta de mortalidade de $11 \%$ nos pacientes intensivamente tratados em relação ao grupo convencional. Os pacientes que não usavam insulina e que tinham perfil de menor risco cardiovascular beneficiaram-se mais (redução de risco de $15 \%$ ), o que foi atribuído ao menor tempo de DM e/ou doença aterosclerótica menos avançada, de forma que o tratamento mais precoce determinou maior benefício.é possível que o uso de insulina agudamente tenha melhorado as condições do miocárdio infartado e não infartado, reduzindo os riscos de mortalidade a curto prazo; no entanto, a mortalidade intra-hospitalar e 3 meses após a alta não foi significativamente menor no grupo intensivamente tratado. Durante o período de seguimento, a glico-hemoglobina manteve-se em níveis mais baixos nos dois grupos, mas foi sempre menor no que recebeu insulina inicialmente e que manteve tratamento intensivo (8). Portanto, a longo prazo, a obtenção de taxas reduzidas de mortalidade cardiovascular pode vir ao encontro dos resultados dos estudos UKPDS e DCCT, nos quais o controle metabólico intensivo, quer seja com drogas ou insulina, mas obtendo menores valores de glico-hemoglobina, determinou redução no risco de eventos cardiovasculares, apesar de significância estatística limítrofe $(9,10)$.

Em pós-operatório de cirurgia cardíaca, a solução de GIK foi usada pela primeira vez por Baimbridge e cols. em pacientes com baixo débito após troca de válvula mitral que não respondiam à abordagem terapêutica usual (11). A partir de então, tanto o número de estudos como o de inclusão de pacientes foram reduzidos, poucos destes randomizados, além de desenhos e desfechos variados. A solução de GIK era utilizada antes, durante e após a cirurgia, a duração de infusão variava de alguns segundos (bolus no circuito do bypass cardiopulmonar), até $18 \mathrm{~h}$, com dose total de glicose, insulina e potássio muito diferentes entre os estudos (12). Todas estas diferenças não permitiram conclusões definitivas a respeito do uso de GIK no pósoperatório de cirurgia cardíaca.

No entanto, o estudo realizado na cidade de Leuven, com indivíduos criticamente doentes de uma unidade de tratamento intensivo, veio a confirmar os resultados benéficos do uso de infusão contínua de insulina e glicose nestes pacientes. Por características próprias da unidade de tratamento intensivo em que foi realizado este estudo, $63 \%$ destes pacientes estava em pós-operatório de cirurgia cardíaca, o que permite que estes resultados sejam extrapolados para esta população. O estudo comparou um grupo de pacientes que foi intensivamente tratado com insulina e glicose, objetivando uma glicemia mantida entre $80-110 \mathrm{mg} / \mathrm{dl}$, com o grupo tradicionalmente tratado, que recebia infusão de insulina e glicose quando suas glicemias excediam $215 \mathrm{mg} / \mathrm{dl}$, com o objetivo de mantê-las entre $180-200 \mathrm{mg} / \mathrm{dl}$. O grupo intensivamente tratado apresentou redução no risco de mortalidade intrahospitalar de $34 \%$. Se estes benefícios se deveram à prevenção da hiperglicemia, à maior disponibilidade de insulina ou a ambos, não se sabe. A principal causa da diminuição da mortalidade nestes pacientes foi atribuída à redução da frequiência de falência de múltiplos órgãos por septicemia. Além disso, a redução da morbidade foi relacionada à redução dos episódios de septicemia, de insuficiência renal aguda, do número de transfusões de sangue, do tempo de ventilação mecânica, da duração 
da internação e da prevalência de polineuropatia. Naturalmente que o grupo que recebeu tratamento intensivo apresentou maior número de episódios de hipoglicemia, embora estes não tenham sido acompanhados por eventos adversos graves, uma vez que o algoritmo para alteração da dose de insulina de acordo com o teste glicêmico garantia sua rápida detecção e correção (13). Alcançar os valores de glicemia próximos de $110 \mathrm{mg} / \mathrm{dL}$ foi necessário para atingir a redução ótima de mortalidade observada, o que sobrepujou, naturalmente, o risco de hipoglicemias (14). A avaliação de custo-benefício foi realizada com os dados do estudo DIGAMI e do estudo de Leuven, e ambas as análises favoreceram o uso de insulina e glicose do ponto de vista econômico (15).

No entanto, apesar de muitos anos terem se passado, não se estabeleceu rotineiramente o uso de soluções de insulina, pela ausência de estudos randomizados de grande porte, entendimento precário dos mecanismos pelos quais seriam efetivas, complexidade para administrá-las e principalmente a mudança cultural que é exigida dos profissionais de saúde para aplicá-las na sua prática. Dentro deste contexto, mais de um ensaio clínico randomizado de grande porte vem sendo conduzido, além de estudos experimentais que vêm sendo publicados recentemente avaliando de forma mais aprofundada os possíveis mecanismos de ação da insulina e glicose nestas situações.

\section{Possíveis Mecanismos da Cardioproteção Pelo Uso de Infusões de Insulina-Glicose}

A descoberta da insulina, seu papel na patogênese do DM e a importância do seu tratamento renderam o prêmio Nobel a Banting e McLeod com seus estudos em cães em 1923. Classicamente são conhecidos os efeitos da insulina sobre o metabolismo dos substratos, mas este hormônio também tem efeitos sobre a função nervosa, hemostasia, metabolismo das lipoproteínas e função vascular. A insulina tem efeitos no coração sobre a utilização de substratos pelos cardiomiócitos $(16,17)$ e também efeitos independentes destes sobre o fluxo coronariano (18), como anti-inflamatório (19) ou, como mais recentemente se propõe, efeitos diretos na sobrevida celular miocárdica $(20,21)$.

\section{Mecanismos da Cardioproteção Pela Insulina: Efeitos Sobre o Metabolismo dos Substratos}

No coração com oxigenação normal, os AGL são a principal fonte de energia dos cardiomiócitos. Sua captação e oxidação são proporcionais às suas concentrações séricas. $\mathrm{Na}$ isquemia miocárdica, a energia celular dependerá quase que exclusivamente do metabolismo anaeróbico da glicose, pois a redução na oferta de oxigênio torna o miocárdio incapaz de oxidar os AGL (22). Um aumento da glicólise e oxidação da glicose dependem da ocorrência de um incremento do transporte deste substrato para a célula miocárdica e ativação da glicogenólise, de forma que a maior captação de glicose e seu metabolismo durante isquemia miocárdica aguda associam-se à preservação da função miocárdica (16).

Até $50 \%$ dos pacientes com IAM apresentam hiperglicemia na admissão hospitalar secundária ao estresse e conseqüente aumento de cortisol e noradrenalina, ou ainda por diabetes sem diagnóstico prévio (23). A hiperglicemia neste momento é fator de pior prognóstico, associando-se a maior risco de insuficiência cardíaca e mortalidade, independente de diagnóstico prévio de diabetes (24-26). Em caso da disponibilidade de glicose estar prejudicada em condições de isquemia, poderá ocorrer prejuízo da função miocárdica. A deficiência de insulina, absoluta ou relativa, característica do DM, leva à menor translocação de GLUT 4 para a membrana celular, limitando a disponibilidade de glicose, o que determina a utilização de AGL pelo miocárdio. Este padrão alterado de utilização de substratos resulta em menor produção de ATP, geração de radicais livres de $\mathrm{O}_{2}$, maior consumo de $\mathrm{O}_{2}$ pelo miocárdio e disfunção contrátil miocárdica (27).

Uma forma de otimizar a utilização de glicose e reduzir a oxidação de AGL é o uso de infusões de glicose e insulina, o que foi o suporte teórico do estudo DIGAMI e tantos outros envolvendo este tipo de terapêutica pós-infarto (7).

Outra forma terapêutica visando o mesmo objetivo é o uso de inibidores pFOX, ranolazina e trimetazidina, que são drogas que inibem parcialmente a oxidação de AGL. A inibição parcial da oxidação de AGL aumenta a utilização de glicose e piruvato pelas células miocárdicas, diminuindo a produção de lactato, resultando em $\mathrm{pH}$ maior e melhor função contrátil durante a isquemia. Diferentemente dos agentes anti-anginosos clássicos, estas drogas não afetam a freqüência cardíaca, fluxo sangüíneo coronariano ou pressão arterial (28). Ensaios clínicos utilizando trimetazidina e ranolazina, sozinhos ou em combinação com bloqueadores do canal de cálcio ou beta-bloqueadores, demonstraram que têm efeito anti-anginoso, sendo úteis para pacientes com angina persistente, apesar de tratamento adequado com as drogas anti-anginosas clássicas $(29,30)$. No entanto, o uso destas estratégias 
alternativas com “moduladores metabólicos" não incorpora os benefícios específicos da insulina durante a síndrome coronariana aguda.

\section{Mecanismos da Cardioproteção Pela Insulina:} Efeitos Sobre a Vasodilatação Coronariana

A resistência à insulina tem sido classicamente considerada uma pior resposta da captação de glicose insulino-mediada por células envolvidas no metabolismo dos substratos (adiposas, hepáticas, de músculo esquelético). No entanto, a resistência à insulina pode envolver qualquer um dos seus efeitos biológicos. Em indivíduos saudáveis, a insulina aumenta o fluxo e volume sangüíneos no músculo esquelético, o que lhe confere características de um hormônio vasodilatador (31). $\mathrm{Na}$ vasculatura periférica, a insulina causa vasodilatação dose e tempodependente, efeito este que está prejudicado em estados caracterizados por resistência à insulina, tais como a obesidade, hipertensão arterial e diabetes. Esta resistência vascular à ação da insulina pode ser um mediador na fisiopatologia vascular (32).

Estudos em animais demonstraram que a insulina aumenta (33) ou não modifica (34) o fluxo sangüíneo miocárdico. Em humanos, a maioria dos estudos concorda com um efeito da insulina em aumentar o fluxo sangüíneo miocárdico (18). Como a insulina é um vasodilatador lento e a vasodilatação promovida é dose- e tempo-dependente, a observação de aumento do fluxo sangüíneo miocárdico com injeções de insulina em bolo não ocorre, havendo a necessidade nestes estudos de utilizar infusão contínua do hormônio, analisando as diferentes respostas de acordo com as doses utilizadas (35).

$\mathrm{Na}$ vasculatura periférica, a insulina causa aumento rápido e dose-dependente da produção de óxido nítrico (NO) pelas células endoteliais (36), além de estimular a Na-K-ATPase, causando hiperpolarização de células musculares lisas, redução de cálcio intracelular e daí relaxamento destas células (37). O efeito da insulina em aumentar a atividade simpática via sistema nervoso central (38) opõe-se ao seu efeito vasodilatador, endotélio-dependente, e o efeito final (vasodilatação) é um balanço entre o vasodilatador endotélio-dependente e vasoconstritor simpático-dependente, motivo pelo qual a insulina é um vasodilatador lento.

Hipertensão e diabetes, importantes fatores de risco cardiovascular, relacionam-se a dano ao endotélio, o que levaria à menor produção de fatores vasodilatadores e maior liberação de fatores vasoconstritores derivados do endotélio. Como a insulina é um fator que causa predominantemente vasodilatação dependente do endotélio, indivíduos com disfunção endotelial teriam resposta reduzida à sua ação. Esta resistência à insulina em nível vascular seria causada pelo desequilíbrio entre produção de fatores vasodilatadores e vasoconstritores derivados do endotélio e estaria envolvida na gênese do processo aterosclerótico (18). No entanto, estudo em indivíduos com diabetes caracteristicamente resistentes à insulina, demonstraram respostas de aumento de fluxo coronariano à injeção de insulina em diferentes doses, semelhantes às de indivíduos normais (35). Já no estudo com indivíduos obesos, foi demonstrada menor resposta vasodilatadora à insulina no músculo cardíaco (39).

\section{Mecanismos da Cardioproteção Pela Insulina: Efeitos Anti-Inflamatórios}

Indivíduos criticamente doentes podem apresentar uma "tempestade" de citocinas, caracterizada pela liberação aguda destas substâncias. O TNF- $\alpha$ é uma citocina pró-inflamatória que também é liberada após o IAM, causando disfunção endotelial, além de determinar atividade pró-coagulante e deposição de fibrina (40).O fator inibidor de macrófagos é liberado na septicemia por bactérias Gram positivas e negativas; sua neutralização pode proteger de endotoxemia e choque tóxico. $\mathrm{O}$ TNF- $\alpha$ pode inibir a ação vasodilatadora da insulina (41) e o fator inibidor de macrófagos pode ser suprimido pela insulina (42), de forma que estes efeitos também devem ser considerados nos mecanismos do benefício do uso de insulina e glicose em indivíduos criticamente doentes.

Estudos recentes demonstraram que a insulina exerce efeitos anti-inflamatórios em células endoteliais de aorta de humanos in vitro (43) e em células mononucleares in vivo. Nestes estudos, a infusão de insulina causou supressão da geração de espécies reativas de oxigênio pelas células mononucleares, com redução plasmática concomitante de proteína $\mathrm{C}$ reativa (PCR), ICAM-1, MCP-1 e PAI-1 (44). A ação da insulina parece ser particularmente relevante quanto à ruptura da placa aterosclerótica, já que neste caso as metaloproteinases têm papel central e são suprimidas especificamente por ela (45). Além disto, fatores teciduais e PAI-I, também suprimidos pela ação da insulina, são importantes no início da trombose e fibrinólise, respectivamente (44).

Considerando que a PCR, proteína de fase aguda sintetizada pelo fígado, serve como indicador da presença de inflamação e necrose tecidual, esta foi avaliada nos pacientes que participaram do estudo de Leuven. Observou-se redução progressiva e mais precoce da PCR com o uso de insulina, diferentemente do grupo que recebeu tratamento convencional, 
sugerindo papel anti-inflamatório daquele tratamento como um dos mecanismos para a redução de mortalidade nestes pacientes. Além disto, níveis reduzidos de lecitina manose-ligada (MBLA), proteína ligada à defesa do hospedeiro e imunidade inata, observados inicialmente nestes indivíduos criticamente doentes, podem ser revertidos pelo tratamento com insulina (19). É possível que a supressão da PCR possa ser parcialmente responsável pelos efeitos vantajosos do tratamento com insulina em indivíduos criticamente doentes e naqueles após IAM.

\section{Mecanismos da Cardioproteção Pela Insulina: Efeitos Anti-Apoptóticos}

Sabe-se que a reperfusão é uma condição fundamental para salvar o tecido miocárdico após um IAM, melhorando a função ventricular e a sobrevida após este evento. No entanto, a reperfusão induz perda celular, além daquela esperada pela isquemia por si só; além da necrose, um componente da morte celular não previamente reconhecido na lesão após reperfusão, denominado morte celular programada ou apoptose, teria um papel biologicamente significativo (46).

Os efeitos da insulina sobre a lesão miocárdica após isquemia-reperfusão foram estudados em ratos anestesiados e no modelo do coração isolado, demonstrando-se benéfico por reduzir a área de infarto, desde que iniciada no início da reperfusão (47). Além disto, o efeito protetor do miocárdio conferido pela insulina ocorreu utilizando glicose ou piruvato como substrato na preparação, de forma que o efeito das infusões de insulina poderia ser, pelo menos em parte, dependente de efeitos da insulina mais do que do substrato utilizado concomitantemente (20). Estes resultados questionam a exclusividade da hipótese metabólica quanto aos efeitos cardioprotetores do GIK e implicam num mecanismo cardioprotetor dependente da insulina e independente da captação de glicose induzida por esta. O estudo das vias de sinalização intra-celular da insulina e sua relação com a redução da área de infarto durante reperfusão veio contribuir ainda mais para o entendimento dos mecanismos benéficos da insulina neste cenário. A insulina exerce suas várias ações pela indução da fosforilação de seu receptor na superfície celular, tornando-o ativo, o que leva à fosforilação de uma série de proteínas do citosol e proteínas ligadas à membrana, o que atua como mediador de suas ações (48). Na figura 1 , pode-se observar uma visão esquemática das vias de sinalização da insulina que promovem sobrevida celular e facilitam a captação de glicose. A insulina parece conferir efeitos anti-apoptóticos pela ativação de vias de sobrevida celular como demonstrado na figura.

A utilização de inibidores farmacológicos das vias de sinalização da insulina demonstrou que a sinalização da tirosina quinase, PI3 quinase e p70S6 quinase são necessárias para que ocorra a cardioproteção. Em conjunto, a atividade dos intermediários promotores de sobrevida celular (proteína quinase B ou Akt, e S6K) é induzida pela administração de insulina durante a reperfusão. Além

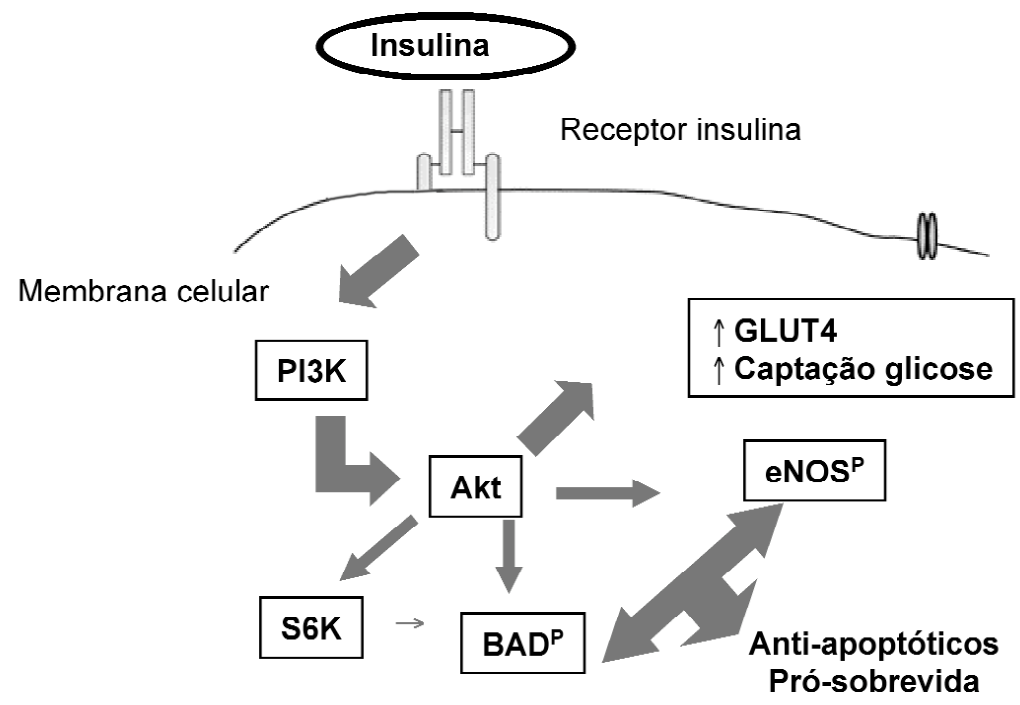

Figura 1. Visão esquemática das vias de sinalização da insulina que promovem sobrevida celular e facilitam a captação de glicose. Akt: proteína quinase B; BAD: Bcl-2/Bcl-Xl-agonista causando morte celular; PI3K: fosfatidilinositol 3,4,5trifosfato; S6K: p70S6 quinase; O p superescrito denota o estado fosforilado de BAD e eNOS. 
disto, foi demonstrado que o tratamento com insulina durante a reperfusão mantém o peptídeo próapoptótico BAD em forma inativa (fosforilado) (20).

Outra via descrita é uma cascata sinalizadora adicional (fosforilação da NO-sintase endotelial), como alvo da PI3-quinase e AKT ativada pela insulina. $\mathrm{O} \mathrm{NO}$ em altas concentrações aumentaria a apoptose celular, enquanto que sua administração em concentrações farmacológicas a reduziria $(49,50)$. Por outro lado, conforme descrito inicialmente, a insulina pode exercer parte de seu efeito vasodilatador através de mecanismo endotélio-dependente, mediado pelo NO. Vários estudos têm demonstrado que a insulina causa fosforilação da $\mathrm{NO}$-sintase endotelial, o que resulta em liberação de NO endotelial dependente de vias PI3k-Akt (5l). Utilizando ratos que foram submetidos à isquemia-reperfusão, recentemente foi observado que a NO-sintase endotelial é fosforilada quando da adição de insulina durante a reperfusão, enquanto que a wortmanina abole o efeito provocado pela insulina. Isto demonstra que a insulina exerce seus efeitos anti-apoptóticos através da via Akt-PI3k, e que a fosforilação da NO-sintase endotelial e conseqüente produção de $\mathrm{NO}$ são importantes efetores que contribuem para o seu efeito cardioprotetor na isquemia/reperfusão miocárdica (21).

Em conjunto, estes dados suportam um papel da insulina em promover sobrevida celular durante a reperfusão via múltiplos eventos sinalizadores prósobrevida e anti-apoptóticos. Estes dados reforçam aqueles do estudo ECLA e da meta-análise de estudos utilizando GIK em pacientes com IAM. No entanto, o modelo do coração isolado tem suas limitações (a reperfusão pós-isquemia pode ser mantida apenas por algumas horas, um tempo que impede o coração de alcançar estado steady-state de viabilidade celular).

\section{Proposta de um Protocolo Prático Para a Infusão de Insulina Contínua}

Baseados nos benefícios do controle glicêmico alcançado pelo uso de infusão contínua de insulina no cenário de pacientes criticamente doentes, após infarto e cirurgias cardíacas, tornou-se fundamental a construção de um protocolo adaptado de outros já existentes na literatura $(13,52-56)$ e que fosse exeqüível na nossa realidade.

Inicialmente, a primeira questão que surge é quais seriam os pacientes potencialmente beneficiados pelo tratamento com infusão de insulina contínua objetivando-se níveis de glicemia normais. Os autores sugerem que seu uso seja considerado em pacientes com e sem diabetes após infarto agudo do miocárdio, após cirurgias cardíacas e em indivíduos criticamente doentes, embora as evidências que suportem estas indicações sejam consideradas graus C e D, embasadas, respectivamente, por investigações randomizadas, controladas, mas com pequeno número de pacientes e não randomizadas, controladas, mas com grande número de pacientes. Considerando-se que o estudo de Leuven contemplava uma amostra de $63 \%$ de pacientes em pós-operatório de cirurgia cardíaca e que os demais estudos tenham contado com indivíduos após infarto com amostras pequenas de pacientes, acreditamos que estes dados, bem como o entendimento dos mecanismos fisiopatológicos da hiperglicemia e os efeitos benéficos do controle da glicemia com insulinoterapia contínua, possam ser extrapolados às populações citadas.

A segunda questão seria a uniformidade de condutas de uma mesma equipe quanto ao controle adequado e rigoroso da glicemia destes pacientes. Para isto, a proposta dos autores é apresentar um protocolo de ajuste de insulinoterapia contínua, o qual deverá estar baseado não somente no nível de glicemia, mas também na velocidade de alteração da glicose no sangue e na sensibilidade à insulina. Por exemplo, em pacientes com glicose sangüínea que apresentam redução de 200 para $130 \mathrm{mg} / \mathrm{dL}$ em $\mathrm{l}$ hora (muito sensíveis à insulina), a velocidade de infusão deverá ser reduzida para prevenir hipoglicemia na hora subseqüente (54).

Embora não existam estudos controlados examinando a freqüência ideal para a realização da monitorização da glicose capilar durante a infusão de insulina contínua, a literatura tem recomendado o controle horário da glicose, permitindo desta forma $\mathrm{o}$ pronto ajuste da infusão de insulina às alterações dos níveis da glicose sangüínea $(55,57,58)$. No entanto, alguns estudos têm sugerido que, após 3 valores de glicemias estáveis dentro do nível desejado, a monito-rização poderia ter um intervalo de 2 a 4 horas $(13,55)$.

A infusão de insulina contínua deverá ser preparada em solução fisiológica a $0,9 \%(13,14,55)$, permanecendo estável por 24 horas à temperatura ambiente ou refrigerada. No entanto, há relatos de que a adsorção desta aos frascos e sistema de infusão é significativa em soluções com menos de 100 a 200UI/L. A adsorção máxima relatada ocorre em 30 a 60 minutos e a quantidade adsorvida no sistema de infusão pode variar de 10 a 80\%, dependendo de vários fatores como concentração de insulina, área da superfície do frasco, volume da solução, tipo e comprimento do equipo, temperatura, presença de albumina, sangue total e eletrólitos, além de alguns 
medicamentos. O percentual de adsorção de insulina é inversamente proporcional à sua concentração na solução. Portanto, para minimizar estes problemas, recomenda-se preencher o equipo e desprezar $50 \mathrm{ml}$ da solução preparada, quando do primeiro uso do equipo, com o objetivo de saturar os locais de ligação no frasco $(53,59)$. Embora não exista referência na literatura que indique a troca da solução de insulina contínua a cada 6 horas, algumas instituições têm seguido esta prática sistematicamente, porém, mais importante do que a troca da infusão de insulinoterapia, é, a cada hora, mobilizar o frasco da solução para reduzir a taxa de adsorção.

A utilização de um protocolo de insulinoterapia contínua guiado por enfermeiros tem como potenciais vantagens a uniformidade de condutas dentro da mesma equipe, evitando, desta forma, ajustes aleatórios e inconsistentes (quadros I, II e III).

\section{Consideraçōes Finais}

É provável que todos os mecanismos aventados acima contribuam, pelo menos parcialmente, na cardioproteção mediada pela insulina e glicose. No entanto, as infusões de insulina e glicose não são utilizadas com a freqüência desejada na prática clínica, por alguns motivos a se considerar: 1) Não há protocolo padrão para o uso destas soluções; 2) Embora exista a falsa impressão de que este tipo de tratamento possa acarretar mais custos, considerandose que existe a necessidade do uso de bomba de infusão e testes glicêmicos freqüentes, pelo menos dois estudos

Quadro 1. Início da infusão.

\begin{tabular}{|c|c|c|c|c|c|c|c|}
\hline \multicolumn{8}{|c|}{ NIVEIS GLICEMICOS (mg/dL) } \\
\hline & 111-162 & 163-209 & 210-252 & 254-306 & 308-361 & $362-432$ & $>432$ \\
\hline Administrar: & IU IV & $2 \mathrm{U} I \mathrm{~V}$ & 3 U IV & $6 \mathrm{U}$ IV & $8 \mathrm{U} \mathrm{IV}$ & $10 \mathrm{U}$ IV & COM \\
\hline Iniciar a: & $1 \mathrm{U} / \mathrm{h}$ & $1 \mathrm{U} / \mathrm{h}$ & $2 \mathrm{U} / \mathrm{h}$ & $2 \mathrm{U} / \mathrm{h}$ & $2 \mathrm{U} / \mathrm{h}$ & $2 \mathrm{U} / \mathrm{h}$ & \\
\hline
\end{tabular}

Quadro 2. Infusão em andamento (abaixo da taxa desejada de $110 \mathrm{mg} / \mathrm{dL}$ ).

\begin{tabular}{|c|c|c|c|c|c|c|}
\hline $\begin{array}{l}\text { Níveis } \\
\text { glicêmicos } \\
\text { (mg/dL) }\end{array}$ & $1-3 \mathrm{U} / \mathrm{h}$ & $4-6 \mathrm{U} / \mathrm{h}$ & 7-9U/h & $10-12 \mathrm{U} / \mathrm{h}$ & $13-16 \mathrm{U} / \mathrm{h}$ & $>16 U / h$ \\
\hline 63 & \multicolumn{6}{|c|}{ desligar infusão e administrar 1 ampola de glicose $50 \%$ IV } \\
\hline 64-81 & \multicolumn{2}{|c|}{$\begin{array}{l}\text { desligar infusão, refazer o teste } \\
\text { glicêmico em } 1 \text { h e se }>126 \mathrm{mg} / \mathrm{dL} \text {, } \\
\text { reiniciar a } 1 \mathrm{U} / \mathrm{h}\end{array}$} & \multicolumn{2}{|c|}{$\begin{array}{l}\text { desligar infusão, refazer o teste } \\
\text { glicêmico em ih e se >, } \\
126 \mathrm{mg} / \mathrm{dL} \text { reiniciar a } 2 U / \mathrm{h}\end{array}$} & \multicolumn{2}{|c|}{$\begin{array}{l}\text { desligar infusão, refazer o teste } \\
\text { glicêmico em lh e se }>126 \mathrm{mg} / \mathrm{dL} \text {, } \\
\text { reiniciar a } 3 \mathrm{U} / \mathrm{h}\end{array}$} \\
\hline $82-99$ & $\begin{array}{l}\text { desligar infusão, } \\
\text { refazer o teste } \\
\text { glicêmico em } 1 \mathrm{~h} \\
\text { e se }>110 \mathrm{mg} / \mathrm{dL} \text {, } \\
\text { reiniciar, mas dimi- } \\
\text { nuir em } 1 \mathrm{U} / \mathrm{h} \text { (da } \\
\text { dose que foi } \\
\text { fechada) }\end{array}$ & & \multicolumn{3}{|c|}{ diminuir a infusão pela metade (em 50\%) } & \\
\hline $100-110$ & \multicolumn{6}{|c|}{ manter a infusão inalterada } \\
\hline $111-126$ & $\begin{array}{l}\Downarrow \text { infusão } \\
\text { em } 1 U / h\end{array}$ & $\begin{array}{l}\Downarrow \text { infusão } \\
\text { em } 2 U / h\end{array}$ & $\begin{array}{l}\Downarrow \text { infusão } \\
\text { em } 3 U / h\end{array}$ & $\begin{array}{l}\Downarrow \text { infusão } \\
\text { em } 4 U / h\end{array}$ & $\begin{array}{l}\Downarrow \text { infusão } \\
\text { em } 5 U / h\end{array}$ & $\begin{array}{l}\Downarrow \text { infusão } \\
\text { em } 6 U / h\end{array}$ \\
\hline
\end{tabular}

\begin{tabular}{|c|c|c|c|c|}
\hline $\begin{array}{l}\text { Níveis } \\
\text { glicêmicos } \\
\text { (mg/dL) }\end{array}$ & $1-5 \mathrm{U} / \mathrm{h}$ & 6-10U/h & $11-16 \mathrm{U} / \mathrm{h}$ & $>16 U / h$ \\
\hline $111-162$ & $\begin{array}{l}\text { administrar IU IV e } \\
\Uparrow \text { infusão em IU/h }\end{array}$ & $\begin{array}{l}\text { administrar } 2 \mathrm{U} \text { IV e } \\
\Uparrow \text { infusão em } 1 \mathrm{U} / \mathrm{h}\end{array}$ & $\begin{array}{l}\text { administrar } 2 \mathrm{U} \text { IV e } \\
\Uparrow \text { infusão em } 2 \mathrm{U} / \mathrm{h}\end{array}$ & \\
\hline $163-209$ & $\begin{array}{l}\text { administrar } 2 \text { U IV e } \\
\Uparrow \text { infusão em } 1 \mathrm{U} / \mathrm{h}\end{array}$ & $\begin{array}{l}\text { administrar } 2 U \text { IV e } \\
\Uparrow \text { infusão em } 2 U / h\end{array}$ & $\begin{array}{l}\text { administrar } 2 \mathrm{U} \text { IV e } \\
\Uparrow \text { infusão em } 3 \mathrm{U} / \mathrm{h}\end{array}$ & \\
\hline $210-252$ & $\begin{array}{l}\text { administrar } 2 \text { U IV e } \\
\Uparrow \text { infusão em } 1 \mathrm{U} / \mathrm{h}\end{array}$ & $\begin{array}{l}\text { administrar } 3 U \text { IV e } \\
\Uparrow \text { infusão em } 2 U / h\end{array}$ & $\begin{array}{l}\text { administrar } 3 U \text { IV e } \\
\Uparrow \text { infusão em } 3 U / h\end{array}$ & avisar plantão \\
\hline $254-306$ & $\begin{array}{l}\text { administrar } 3 \mathrm{U} \text { IV e } \\
\Uparrow \text { infusão em } 1 \mathrm{U} / \mathrm{h}\end{array}$ & $\begin{array}{l}\text { administrar } 5 \mathrm{U} \text { IV e } \\
\Uparrow \text { infusão em } 2 \mathrm{U} / \mathrm{h}\end{array}$ & $\begin{array}{l}\text { administrar } 5 \mathrm{U} \text { IV e } \\
\Uparrow \text { infusão em } 3 \mathrm{U} / \mathrm{h}\end{array}$ & \\
\hline $308-361$ & $\begin{array}{l}\text { administrar } 8 \mathrm{U} \text { IV e } \\
\Uparrow \text { infusão em } 1 \mathrm{U} / \mathrm{h}\end{array}$ & $\begin{array}{l}\text { administrar } 8 \mathrm{U} \text { IV e } \\
\Uparrow \text { infusão em } 2 \mathrm{U} / \mathrm{h}\end{array}$ & $\begin{array}{l}\text { administrar 8U IV e } \\
\Uparrow \text { infusão em } 3 U / h\end{array}$ & \\
\hline $\begin{array}{l}362-432 \\
>432\end{array}$ & $\begin{array}{l}\text { administrar 10U IV e } \\
\Uparrow \text { infusão em 1U/h }\end{array}$ & $\begin{array}{l}\text { administrar } 10 \mathrm{U} \text { IV e } \\
\Uparrow \text { infusão em } 2 \mathrm{U} / \mathrm{h} \\
\text { avisar plantão }\end{array}$ & $\begin{array}{l}\text { administrar 10U IV e } \\
\Uparrow \text { infusão em } 3 \mathrm{U} / \mathrm{h}\end{array}$ & \\
\hline
\end{tabular}

Adaptado de: Brown G. Crit Care 2001;29:1714-19(54). 
demonstraram sua vantagem, quando se considera a morbi-mortalidade reduzida nos pacientes tratados (menor tempo de internação, menor risco de infecção grave); 3) $\mathrm{O}$ estudo em indivíduos criticamente doentes foi realizado em apenas um centro, e a população estudada era predominantemente de pacientes em pós-operatório de cirurgia cardíaca.

Para superar estes entraves, sugere-se nesta revisão um protocolo de infusão contínua de insulina (quadros I, II e III) para uso em indivíduos após infarto do miocárdio, após cirurgias cardíacas e pacientes criticamente doentes, que deve ser adaptado naturalmente à realidade da instituição onde será aplicado. É possível atingir normoglicemia com segurança em 24 h e mantê-la através de um protocolo, no entanto, é necessária mudança cultural e treinamento de toda equipe de saúde envolvida diretamente no cuidado destes pacientes, objetivando o sucesso pleno da rotina implantada. Não há razão para se imaginar que os dados obtidos no estudo de Leuven não possam ser extrapolados para todos os pacientes criticamente doentes.

Finalmente, espera-se que estudos em andamento, tais como o ECLA-GIK II e outros do grupo de Leuven, avaliando subpopulações específicas de pacientes criticamente doentes, possam contribuir para o melhor entendimento dos efeitos da infusão de insulina e glicose, suportando definitivamente sua ampla utilização nas unidades de tratamento intensivo e de pós-operatório.

\section{REFERÊNCIAS}

1. Sodi-Pallares D, Testelli MR, Fishleder BL, Bisteni A, Medrano GA, Friedland $C$, et al. Effects of an intravenous infusion of potassium-insulina-glucose solution on the electrocardiographic signs of myocardial infarction. Am J Cardiol 1962;9:166-81.

2. Opie L. Substrate utilization and glycolysis in the heart. Cardiology 1971;56(1):2-21.

3. Fath-Ordoubadi F, Beatt KJ. Glucose-insulin-potassium therapy for treatment of acute myocardial infarction: an overview of randomized placebo-controlled trials. Circulation 1997;96:1152-6.

4. Diaz R, Paolasso EA, Piegas LS, Leopoldo S, Tajer CD, Moreno $\mathrm{C}$, et al. on behalf of the ECLA Collaborative Group. Metabolic modulation of acute myocardial infarction. The ECLA glucose-insulin-potassium pilot trial. Circulation 1998;98:2227-34.

5. Stanley A, Moraski RE, Russell RO, Rogers WJ, Mantle $J A$, Kreisberg RA, et al. Effects of glucose-insulinpotassium on myocardial substrate availability and utilization in stable coronary artery disease. Studies on myocardial carbohydrate, lipid and oxugen arterial-coronary sinus differences in patients with coronary artery disease. Am J Cardiol 1975;36:929-37.

6. Ceremuzynski L, Budaj A, Czepiel A, Burzykowski T, Achremczyk P, Smielak-Korombel W, et al. Low-dose glucose-insulin-potassium is ineffective in acute myocardial infarction: results of a randomized multicenter Pol-GIK trial. Cardiovasc Drugs Ther 1999;13:191-200.

7. Malmberg K, Ryden L, Efendic S. Randomized trial of insulin-glucose infusion followed by subcutaneous insulin treatment in diabetic patients with acute myocardial infarction (DIGAMI Study): effects on mortality at 1 year. J Am Coll Cardiol 1995;26:57-65.

8. Malmberg K for the DIGAMI (Diabetes Mellitus, Insulin Glucose Infusion in Acute Myocardial Infarction) Study Group. Prospective randomised study of intensive insulin treatment on long term survival after acute myocardial infarction in patients with diabetes mellitus. Br Med J 1997;314:1512-5.

9. UKPDS. Intensive blood-glucose control with sulphonylureas or insulin compared with conventional treatment and risk of complications in patients with type 2 diabetes (UKPDS 33). Lancet 1998;352:837-53.

10. DCCT. The effect of intensive treatment of diabetes on the development and progression of long term complications in insulin-dependent diabetes mellitus. N Engl J Med 1993;329:977-86.

11. Braimbridge M, Clement AJ, Brown AH, Sabar E, Mendel D. Triple starr valve replacement. Br Med J 1969;3(672):683-8.

12. Doenst T BW, Beyersdorf F. Therapy with insulin in cardiac surgery: controversies and possible solutions. Ann Thorac Surg 2003;75(2):S721-S8.

13. Van Den Berghe $G$, Wouters $P$, Weekers $F$, Verwaest $C$, Bruyninckx $F$, Schetz $M$, et al. Intensive insulin therapy in critically ill patients. N Engl J Med 2001;345(19):1359-67.

14. Van Den Berghe G, Wouters P, Bouillon R, Weekers F, Verwaest C, Schetz $M$, et al. Outcome benefit of intensive insulin therapy in the critically ill: Insulin dose versus glycemic control. Crit Care Med 2003;31(2):359-66.

15. Almbrand B, Johannesson M, Sjöstrand B, Malmberg K, Rydén L. Cost-effectiveness of intensive insulin treatment after acute myocardial infarction in patients with diabetes mellitus. Results from the DIGAMI study. Eur Heart J 2000;21:733-9.

16. Zorzano A, Sevilla L, Camps M, Becker C, Meyer J, Kammermeier $\mathrm{H}$, et al. Regulation of glucose transport, and glucose transporters expression and trafficking in the heart: studies in cardiac myocites. Am J Cardiol 1997;80(3A):65A76A.

17. Taegtmeyer $\mathrm{H}$, Passomore JM. Defective energy metabOlism of the heart in diabetes. Lancet 1985;1(8421):139.

18. Sundell J, Knuuti J. Insulin and myocardial blood flow. Cardiovasc Res 2003;57:312-9.

19. Hansen T, Thiel S, Wouters PJ, Christiansen JS, Van Den Berghe $G$. Intensive insulin therapy exerts antiinflamatory effects in critically ill patients and counteracts the 
adverse effect of low mannose-binding lectin levels. J Clin Endocrinol Metab 2003;88:1082-8.

20. Jonassen A, Sack MN, Mjos OD, Yellon DM. Myocardial protection by insulin at reperfusion requires early administration and is mediated via Akt and p70s6 kinase cell-surval signaling. Circ Res 2001;89:1191-8.

21. Gao F, Gao E, Yue TL, Ohlstein EH, Lopez BL, Cristopher TA, et al. Nitric oxide mediates the antiapoptotic effect of insulin in myocardial ischemia-reperfusion: The roles of PI3-kinase, Akt, and endothelial nitric oxide synthase phosphorylation. Circulation 2002;105:1497-502.

22. Opie L. Metabolic response during impending myocardial infarction. I. Relevance of studies of glucose and fatty acid metabolism in animals. Circulation 1972;45:483-90.

23. Norhammar A, Tenerz A, Nilsson G, Hamsten A, Efendic $S$, Rydén L, et al. Glucose metabolism in patients with acute myocardial infarction and no previous diagnosis of diabetes mellitus: a prospective study. Lancet 2002;359:2140-4.

24. Capes S, Hunt D, Malmberg K, Gerstein HC. Stress hyperglycemia and increased risk of death after myocardial infarction in patients with and without diabetes: a systematic overview. Lancet 2000;355:773-8.

25. Malmberg K, Norhammar A, Wedel H, Rydén L. Glycometabolic state at admission: Important risk marker of mortality in conventionally treated patients with diabetes mellitus and acute myocardial infarction. Long term results from the Diabetes and Insulin-Glucose Infusion in Acute Myocardial Infarction (DIGAMI) Study. Circulation 1999;99:2626-32.

26. Wahab N, Cowden EA, Pearce NJ, Gardner MJ, Merry H, Cox JL. Is blood glucose an independent predictor of mortality in acute myocardial infarction in the trombolytic era? J Am Coll Cardiol 2002;40(10):1748-54.

27. Garvey W, Hardin D, Juhaszova M, Dominguez JH. Effects of diabetes on myocardial glucose transport system in rats: implications for diabetic cardiomyopathy. Am J Physiol (Heart Circulation Physiology) 1993;264: $\mathrm{H} 837-\mathrm{H} 44$.

28. Conti C. Partial fatty acid oxidation (pFOX) inhibition: a new therapy for chronic stable angina. Clin Cardiol 2003;26(4):161-2.

29. Pepine C, Wolff AA. A controlled trial with a novel antiischemic agent, ranolazine, in chronic stable angina pectoris that is responsive to conventional antianginal agents. Ranolazine Study Group. Am J Cardiol 1999;84(1):46-50.

30. Detry J, Leclercq PJ. Trimetazidine European Multicenter Study versus propranolol in stable angina pectoris: contribution of Holter electrocardiographic ambulatory monitoring. Am J Cardiol 1995;76(6):8B-11B.

31. Raitakari M, Knuuti MJ, Ruotsalainen U, Laine H, Makea $P$, Teras $M$, et al. Insulin increases blood volume in human skeletal muscle: studies using (150)CO and positron emission tomography. Am J Physiol 1995;269:E1000-E5.

32. Mather K, Anderson TJ, Verma S. Insulin action in the vasculature: physiology and pathophysiology. J Vasc Res 2001;38(5):415-22.

33. Liang C, Doherty JU, Faillace R, Maekawa K, Arnold S, Gavras $\mathrm{H}$, et al. Effects on systemic and coronary hemodynamics, regional blood flows, and plasma cathecolamines. J Clin Invest 1982;69:1321-36.

34. Tune J, Mallet RT, Downey HF. Insulin improves contractile function during moderate ischemia in canine left ventricle. Am J Physiol 1998;274:H1574-H81.

35. Sundell J, Nuutila P, Laine $H$, Luotolahti M, Kaliokoski $K$, Raitakari $O$, et al. Dose-dependent vasodilating effects of insulin on adenosine-stimulated myocardial blood flow. Diabetes 2002;51:1125-30.

36. Zeng G, Quon MJ. Insulin-stimulated production of nitric oxide is inhibited by wortmannin. Direct measurement in vascular endothelial cells. J Clin Invest 1996;98:8948.

37. Tack C, Lutterman JA, Vervoort G, Thien T, Smits P. Activation of the sodium-potassium pump contributes to insulin-induced vasodilation in humans. Hypertension 1996;28:426-32.

38. Anderson E, Hoffman RP, Balon TW, Sinkey CA, Mark AL. Hyperinsulinemia produces both sympathetic neural activation and vasodilation in normal humans. J Clin Invest 1991;87:2246-52.

39. Sundell J, Laine $H$, Luotolahti M. Obesity affects myocardial vasoreactivity and coronary flow response to insulin. Obes Res 2002;10:617-24.

40. Tziakas DN, Chalikias GK, Hatzinikolaou HI, Parissis JT, Papadopoulos ED, Trypsianis $G A$, et al. Anti-inflammatory cytokine profile in acute coronary syndromes: behavior of interleukin-10 in association with serum metalloproteinases and proinflammatory cytokines. Intern J Cardiol 2003;92(2-3):169-75.

41. Aljada A, Ghanim H, Assian E, Dandona P. Tumor necrosis factor-alpha inhibits insulin-induced increase in endothelial nitric oxide synthase and reduces insulin receptor content and phosphorylation in human aortic endothelial cells. Metabolism 2002;51(4):487-91.

42. Das UN. Current advances in sepsis and septic shock with particular emphasis on the role of insulin. Med Sci Monitor 2003;9(8):RA181-92.

43. Aljada A, Ghanim H, Saadeh R, Dandona P. Insulin inhibits NFkB and MCP-1 expression in human aortic endothelial cells through stimulation of nitric oxide. J Clin Endocrinol Metab 2001;86:450-3.

44. Aljada A, Ghanim H, Mohanty P, Kapur N, Dandona P. Insulin inhibits the pro-inflammatory transcription factor early growth response gene-1 (Egr)-1 expression in mononuclear cells (MNC) and reduces plasma tissue factor (TF) and plasminogen activator inhibitor-1 (PAl-1) concentrations. J Clin Endocrinol Metab 200287:141922.

45. Ghanim H, Mohnaty P, Aljada A, Chowhan S, Tripathy D, Dandona $P$. Insulin reduces the pro-inflammatory transcription factor, activation protein-1 (AP-1) in mononuclear cells (MNC) and plasma matrix metalloproteinase-9 (MMP-9) concentration. Diabetes 
2001;50(suppl 2):A408.

46. Fliss $H$, Gattinger D. Apoptosis in ischemic and reperfused rat myocardium. Circ Res 1996;79:949-56.

47. Jonassen A, Aasum E, Riemersma RA, Mjos OD, Larsen TS. Glucose-insulin-potassium reduces infarct size when administered during reperfusion. Cardiovasc Drugs Ther 2000;14(615):623.

48. Carvalheira J, Zecchin HG, Saad MJA. Vias de sinalização da insulina. Arq Bras Endocrinol Metab 2002;46(4):419-25.

49. Rossig L, Haendeler J, Hermann C, Malchow P, Urbich C, Zeiher AM, et al. Nitric oxide down-regulates MKP-3 mRNA levels: involvement in endothelial cell protection from apoptosis. J Biol Chem 2000;275(33):25502-7.

50. Dimmeler S, Haendeler J, Nehls M, Zeiher AM. Suppression of apoptosis by nitric oxide via inhibition of interleukin-1beta-converting enzyme (ICE)-like and cysteine protease protein (CPP)-32-like proteases. J Exp Med 1997;185(4):601-7.

51. Zeng G, Nystrom FH, Ravichandran LV, Cong LN, Kirby $\mathrm{M}$, Mostowski $\mathrm{H}$, et al. Roles for insulin receptor, PI3kinase, and Akt in insulin-signaling pathways related to production of nitric oxide in human vascular endothelial cells. Circulation 2000;101(13):1539-45.

52. Kanji S, Singh A, Tierney M, Meggison H, Mclntyre L, Hebert PC. Standardization of intravenous insulin therapy improves the efficiency and safety of blood glucose control in critically ill adults. Intensive Care Med 2004;30(5):804-10.

53. Goldberg PA, Siegel MD, Sherwin RS, Halickman JI, Lee $M$, Bailey VA, et al. Implementation of a safe and effective insulin infusion protocol in a medical intensive care unit. Diab Care 2004;27(2):461-7.
54. Trence D, Kelly JL, Hirsch IB. The rationale and management of hyperglycemia for in-patients with cardiovascular disease: Time for change. J Clin Endocrinol Metab 2003;88(6):2430-7.

55. Brown G, Dodek P. Intravenous insulin nomogram improves blood glucose control in the critically ill. Crit Care Med 2001;29(9):1714-9.

56. Bonnier M, Lonnroth P, Gudbjornsdottir S, Attvall S, Jansson PA. Validation of a glucose-insulin-potassium infusion algorithm in hospitalized diabetic patients. J Intern Med 2003;253(2): 189-93.

57. Markovitz LJ, Wiechmann RJ, Harris N, Hayden V, Cooper J, Johnson $G$, et al. Description and evaluation of a glycemic management protocol for patients with diabetes undergoing heart surgery. Endocr Pract 2002;8(1):10-8.

58. The Portland Protocol for continuous intravenous insulin infusion in post operative diabetic cardiac surgery patients. www.starrwood.com/research/ insulin/html, 2003.

59. Trissel LA. Handbook of injectable drugs. $11^{a}$ ed. Bethesda (MD): American Society of Hospital Pharmacists, 2001.

\section{Endereço para correspondência:}

Beatriz D. Schaan

Unidade de Pesquisa

Instituto de Cardiologia do Rio Grande do Sul

Av. Princesa Isabel 370

90620-001 Porto Alegre, RS

Fax.: (51) 3230-3600 ramal 3877 / 3927

E-mail: pesquisa@cardiologia.org.br /

beatrizschaan@terra.com.br 